\title{
An Energy Efficient Congestion control Technique for Wireless Sensor Networks
}

\author{
A. Shakeela Joy \\ PG Student, Department of CSE \\ UCEN, AUT, Nagercoil Campus \\ Tamil Nadu, India
}

\author{
M. Muthuselvi \\ Asst.Professor,Department of CSE \\ UCEN, AUT, Nagercoil Campus \\ Tamil Nadu, India
}

\author{
V. Kavitha \\ $\mathrm{PhD}$, Director In-Charge \\ UCEN, AUT, Nagercoil Campus \\ Tamil Nadu, India
}

\begin{abstract}
WSN are distributed networks with small, light weight wireless sensor nodes. These sensor nodes cooperatively monitor the environment to measure the physical parameter such as temperature, pressure etc. It is data centric. When sensor nodes detect an event, it become active in collecting and transmitting the data, which cause congestion and results in packet drops, decrease in throughput and retransmission of data. To ensure the application's reliability requirements and to reduce the load on the network, WSN need proficient congestion control policies. With this aim, this paper evaluates a contextual cooperative congestion control policy that makes use of the environment information of each node to reduce the channel load, by satisfying the applications requirements. This paper proposes a selective data forwarding scheme to avoid congestion and to optimize the energy drain. The SDFS scheme depends on parameters such as available energy and precedence of the data at the node. This scheme collects data from other sensors of this context.
\end{abstract}

\section{Keywords}

Wireless Sensor Network, Congestion control, Contextual Cooperative Mode, Selective Data Forwarding Scheme.

\section{INTRODUCTION}

Wireless sensor networks (WSN) consists of wireless nodes. These nodes are scattered in a decentralized manner to monitor the environment or system. Each node in sensor network consists of three subsystems: the sensor subsystem which senses the environment, the processing subsystem which performs the local computations on the sensed data, and the communication subsystem which is responsible for message exchange with neighboring sensor nodes. Individual sensors have limited sensing region, processing power, and energy. When sensor nodes detect an event, it become active in collecting and transmitting the data, which cause congestion and results in packet drops, decrease in throughput and retransmission of data. Some of the congestion control techniques are TADR [1], DPCC [2] and IPD [3]. In this paper we evaluate a Contextual Cooperative Mode (CCM) [4] to reduce the congestion in the link level. In this mode each sensor node periodically broadcast 1-hop CAM (Cooperative Awareness Messages) through a common channel, referred as communication channel. CAM carries the information such as node energy and node position. The data exchanged through CAMs is used to reduce congestion. By transmitting the important data the congestion can be avoided and the energy level can be consumed. For instance, priority is assigned to the data [5]. We propose an energy efficient algorithm [6] to optimize the energy drain. For this, a Selective Data Forwarding Scheme (SDFS) is used, which transmits the data that has highest precedence, while discarding the data with least precedence [7]. In this scheme the sensor node decision depends on the available energy and precedence of the data at the node.

\section{CAUSES OF CONGESTION}

In WSN congestion occurs due to two main reasons. The first reason for congestion is buffer overflow in the node, ie.when the data arrival rate exceeds the data service rate, which results in congestion on the particular node. The second reason of congestion is contention, interference and the bit error rate on a link, which results in link level congestion. In WSN congestion has a direct impact on energy efficiency and QoS of an application. Congestion cause buffer overflow, that leads to queuing delay, higher packet loss, degrade in link utilization and wastage of energy. Therefore congestion must be controlled efficiently. Congestion control refers to techniques and mechanisms that can prevent congestion before it happens or remove congestion after it has happens by keeping the load below the capacity.

The rest of this paper is organized as follows. Section 3 discusses about the Contextual Cooperative Mode (CCM), Section 4 describes the proposed scheme, Section 5 discusses the simulation scenario, Section 6 discusses the performance evaluation, Section 7 discusses the comparison of congestion control techniques and finally the conclusion in Section 8.

\section{CONTEXTUAL COOPERATIVE MODE(CCM)}

Without sacrificing the application's reliability, sensor nodes operating in this mode use their traffic context information to reduce unnecessary intrusions and the channel load [4]. The operation is based on the periodical broadcast of 1-hop CAMs (Cooperative Awareness Messages) on a common channel, referred as the communication channel. CAM carries the information such as node energy and node position. The data exchanged through CAMs are used to reduce congestion in the link level. Each sensor node uses the specific positions of neighboring sensor node to reconfigure its application requirements and the resulting transmission parameters.

\subsection{Path change assistance application}

Based on the information received from surrounding sensor node by $\mathrm{CAM}$, the path change assistance application provides information to each sensor node [4]. This information states whether the data can be transmitted to the neighbor sensor node in a safe way or not. 
In the path change assistance application, typical situations (one depicted in Figure 1) can be detected and exploited to reduce the channel load.

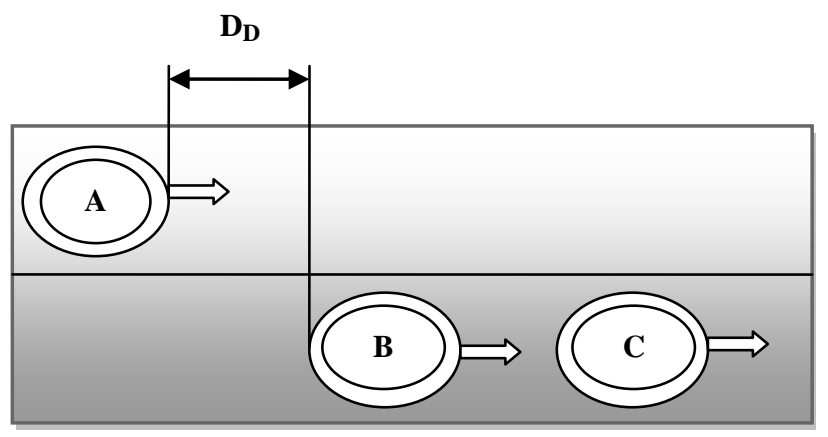

Figure 1: Path change aid scenario

In this sensor node $\mathrm{A}$ knows that the data forwarded to sensor node B is safe. (i.e. sensor node B previously received at least one CAM from sensor node $\mathrm{A}$ ).

\section{$\mathbf{D}_{\mathbf{S}}$}

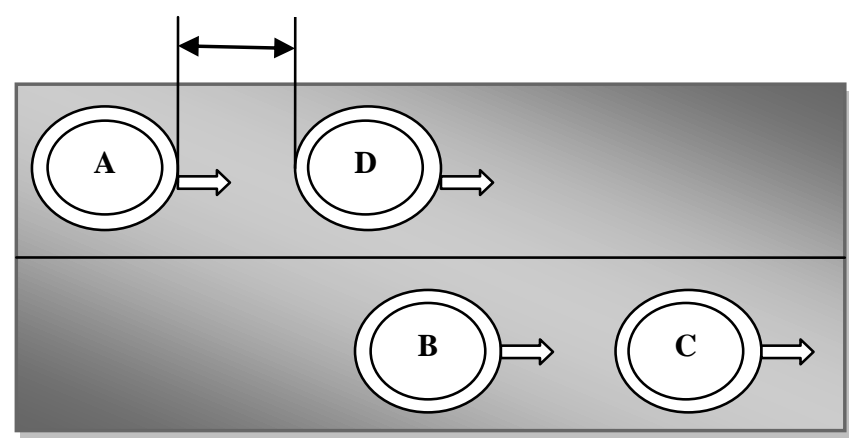

Figure 2: Traffic contextual information In this scenario Figure 2 sensor node $\mathrm{A}$ has to make a decision to forward the data to one of its neighbor sensor node $\mathrm{B}$ or sensor node $\mathrm{D}$. This can be done by calculating $D_{D}$ and $D_{S} . D_{D}$ is defined as the distance between two neighbor sensor nodes in the different path. $\mathrm{D}_{\mathrm{S}}$ is defined as the distance between two neighbor sensor nodes in the same path. The decision $D$ is made by $D=\min \left(D_{D}, D_{S}\right)$. Here $D_{S}<D_{D}$ so the sensor node $A$ transmit the data to the sensor node $\mathrm{D}$. Therefore unnecessary intrusions are reduced and the channel load is decreased. The $\mathrm{D}_{\mathrm{D}}$ and $\mathrm{D}_{\mathrm{S}}$ is calculated by $D_{D}=D_{S}=\operatorname{sqrt}\left(\left(N_{A}-N E_{B}\right)^{2}+\left(N P_{A}-N P_{B}\right)^{2}\right)$. Where NE is the Node Energy and NP is the Node Position of sensor nodes A and $\mathrm{B}$ respectively.

Before calculating D, we should receive at least one CAM to ensure the application's reliability. The probability of receiving at least one CAM is $(\mathrm{Papp}=0.99)$.

\section{SELECTIVE DATA FORWARDING SCHEME (SDFS)}

The main idea in the SDFS is as follows (i) Each sensor node periodically receives 1-hop CAM before detecting an emergency event, (ii) Sensor nodes collects the events data and makes a decision in forwarding the data to one of its neighbors or not, (iii) Estimation of energy is calculated.

The basic architecture concept of SDFS is shown in Figure 3 the SDFS contains Forward Originator (FO) and Forward Data Originator (FDO). In SDFS we assume that each sensor node sends a 1-hop CAM to its neighbors periodically. CAM contains the information such as node energy and node position. When sensor node receives 1-hop CAM from the neighbor sensor nodes, it should check whether the sensor node is behind, it is in the same path or different path. The forward originator (FO) finds the relevant forward node among the neighbor nodes and stores the relevant information in the forward table. When sensor node detects a new event, immediately it collects the data from the environment. The new data is stored in the forward table. In the forward table the data is updated using precedence choice rule. The forward table is shared by both FO and FDO. The available energy in the forward node is estimated based on the energy exhausted during inactive state, data transmission and data reception. Based on the estimation of energy level, the data is either transmitted or discarded.

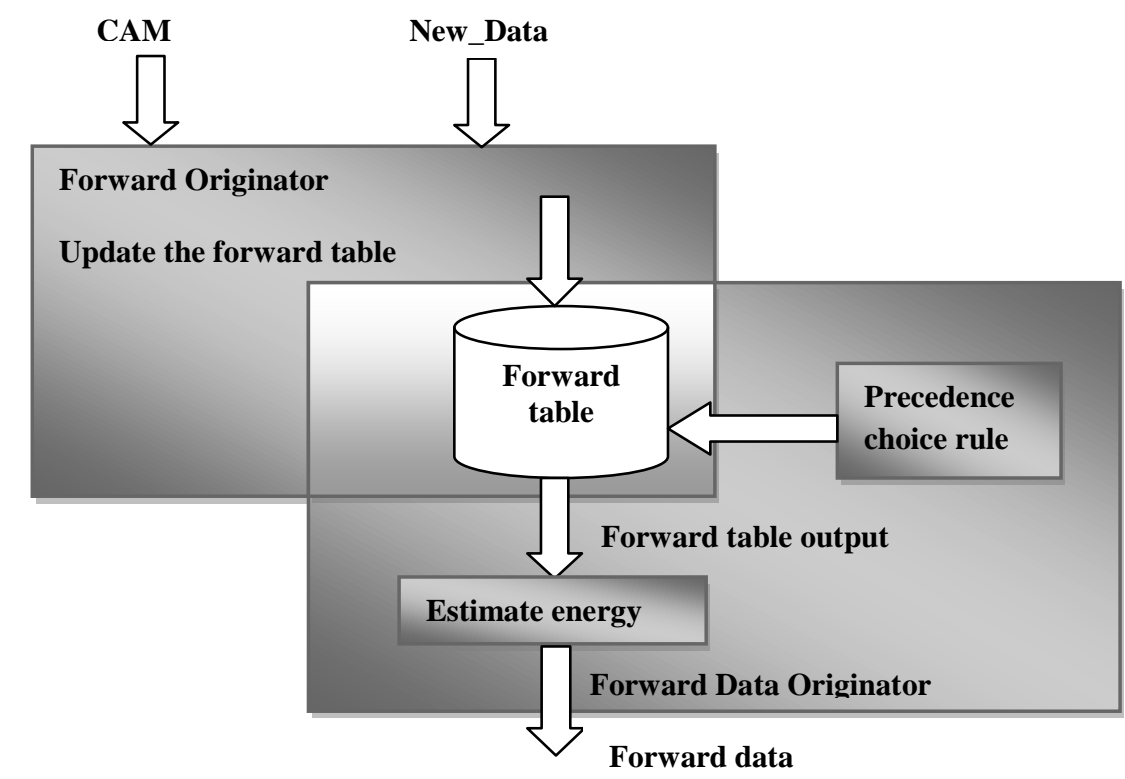

Figure 3: Architecture of SDFS 
In WSN the sensor nodes are randomly distributed. So any sensor node can join and leave the network frequently. Therefore the forward table must be updated periodically at regular intervals. A timestamp is used to detect whether the sensor node receive the 1-hop CAM within the time from the neighbor sensor node or not. If sensor node does not receive any 1-hop CAM from the neighbors before the timestamp exceeds, then it will delete the forward information about the neighbor sensor node from the forward table. The procedure and process of SDFS is given in Table 1 and Figure 4 respectively.

\subsection{Estimation of energy}

Consider a WSN as a collection of Sensor nodes $n_{i}$, where $i=0 \ldots N-1$. Each Sensor node $n_{i}$ has two properties: $e_{t}$ Energy available in the specified node at time $t$ and $x t-$
Important data to be forward at time $t$. We assume that at time $t$, if the node does not receive any data to transmit, then $x_{t}=0$ and if the node receive data to transmit, then $\mathrm{x}_{\mathrm{t}}>0$ [8].

The Dijkstra's algorithm is used for routing. In this algorithm, if any sensor node receives data, it has to take a decision $\mathrm{d}_{\mathrm{t}}$, whether to forward the data to one of its neighbors node or not. If the node decide to forward the data, then $d_{t}=1$ and if the node decides to discard the data, then $d_{t}=0$. The energy in the sensor node decreases due to data reception, transmission and inactive state. It can be represented as follows $\mathbf{E}_{\mathbf{I}}$ : the energy exhausted during inactive state, $\mathbf{E}_{\mathbf{R}}$ : the energy exhausted when receiving or generating a data and $\mathbf{E}_{\mathbf{T}}$ : the energy exhausted when transmitting a data. The available energy at time $t$ can be estimated as follows

$$
e_{t+1}=e_{t}-d_{t}\left(E_{R}+E_{T}\right)-\left(1-d_{t}\right) I_{x t>0} E_{R}-\left(1-d_{t}\right) I_{x t=0} E_{I}
$$

On receiving 1-hop CAM and new data.

\section{Table 1 Procedure for SDFS}

/*Decision whether the data can be forward or not (to the neighbors)*/

/*If the data is not forwarded, discard it*/

$1 *$ otherwise determine the importance of the data by using precedence choice rule*/

/* Estimation of energy is calculated */

01: FOR each New_Data

02: Refer to forward table;

03: IF forward table is empty;

04: Add new data (New_Data) to forward table entry (forward entry);

05: $\quad$ IF available energy $\left(\mathrm{e}_{\mathrm{t}}\right)<$ energy need to transmit a data $\left(\mathrm{E}_{\mathrm{T}}\right)$

06: Discard the data;

07: $\quad$ ELSE

08: $\quad$ Transmit the data;

09: $\quad$ END IF:

10: ELSE /*multiple forward data (Multi_Data) exist in forward entry*/

11: Search for New_Data in forward entry;

12: IF New_Data exist;

13: Replace Ext_Data with new New_Data;

14: $\quad$ IF New_Data Precedence $>$ Ext_Data among the multiple forward data;

15: Delete the one having the less precedence among the multiple forward data;

16: $\quad$ IF energy available $\left(\mathrm{e}_{\mathrm{t}}\right)<$ energy need to transmit a data $\left(\mathrm{E}_{\mathrm{T}}\right)$

17: $\quad$ Discard the data;

18: $\quad$ ELSE

19: $\quad$ Transmit the data;

20: $\quad$ END IF;

21: Restore forward table by precedence choice rule;

22: $\quad$ ELSE

23: $\quad$ Add New_Data to forward entry;

24: $\quad$ Restore forward table by precedence choice rule;

25: $\quad$ END IF;

26: $\quad$ ELSE $/ *$ first time receiving the New_Data*/

27: IF the number of data in the forward entry $<$ Max_Value

28: $\quad$ Add New_Data to the forward entry;

29: $\quad$ Restore forward table by precedence choice rule;

30: $\quad$ ELSE

31: $\quad$ END IF;

33: END IF;

34: END IF;

35: END FOR

/* precedence choice rule $* /$

01:IF New_Data precedence (New-Prece) $>$ Ext_Data precedence

02: Important data to be sent is New_Data;

03:ELSE

04: Temp_Data $=$ New_Data;

05: New_Data $=$ Ext_Data;

06: $\quad$ Ext_Data $=$ Temp_ Data;

07: END IF;

/* forward table $* /$

/* Forward table contain the following information*/ 
01: float Max_value; /* Maximum value in the forward table*/

02: float EA; $\quad / *$ Energy available $\mathrm{e}_{\mathrm{t}}$ is declared as EA */

03: float ET; $/ *$ Energy spent during data transmission $\mathrm{E}_{\mathrm{T}}$ is declared as ET */

04: int priority; $/ *$ Precedence of the data*/

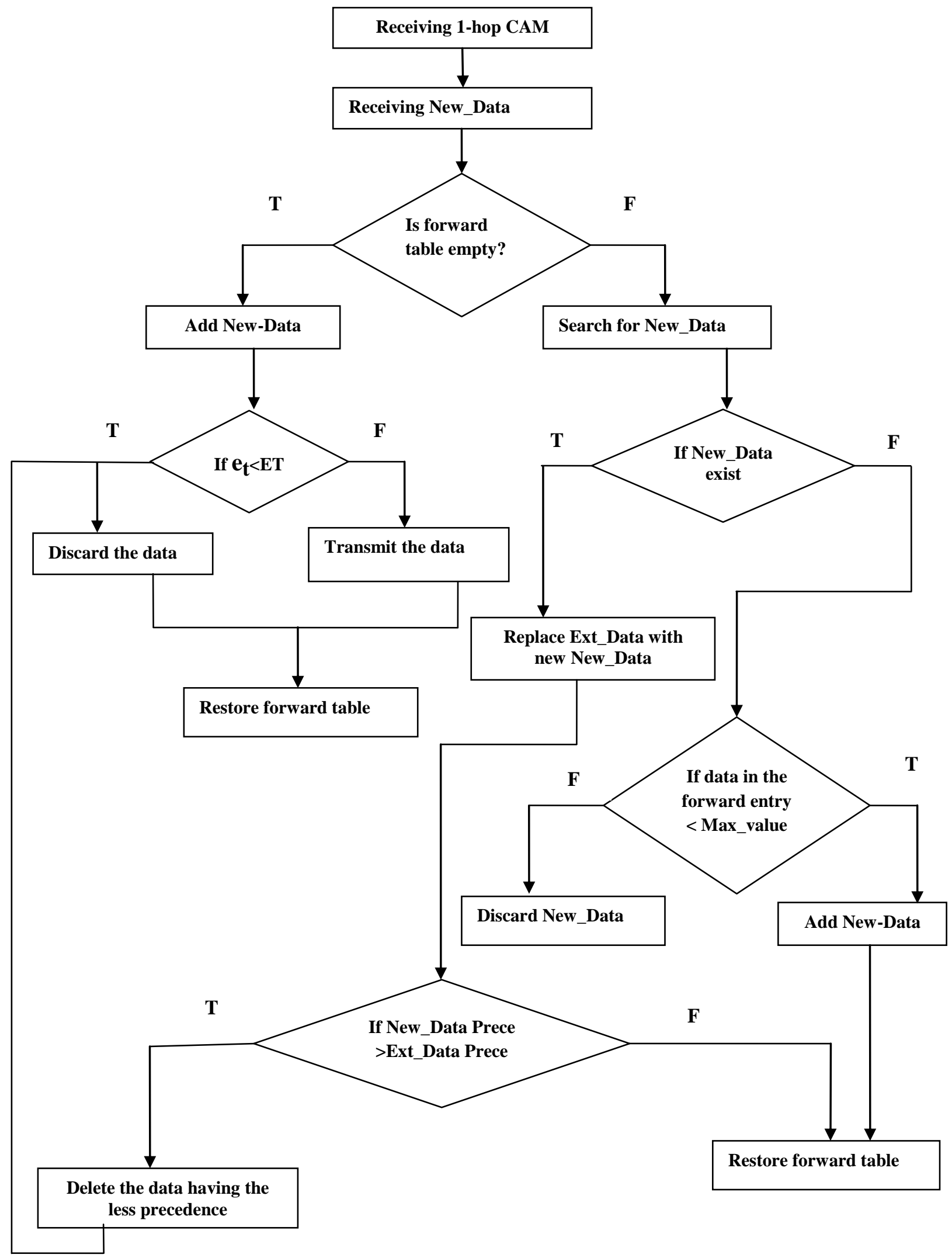

Figure 4: Process of SDFS 


\section{SIMULATION RESULT}

The analysis of the proposed scheme is shown in Figure 5 In scenario 1 , we assume a WSN with ten sensor nodes, data rate is $12 \mathrm{kbps}$, node energy is 20Joule/node, node position is random, and bandwidth is $5 \mathrm{MB}$. If the sensed data is sent to the sink node by the entire sensor node for every $5 \mathrm{~ms}$, then congestion will occur in some sensor nodes. In scenario 2, we consider the WSN Congestion Control Scenario without Energy Efficient and SDFS. Congestion leads to packet loss, delay, energy waste due to packet drops and data retransmission. In scenario 3, we consider the WSN Congestion Control Scenario with Energy Efficient and SDFS. Congestion control in WSN increases the network performance.

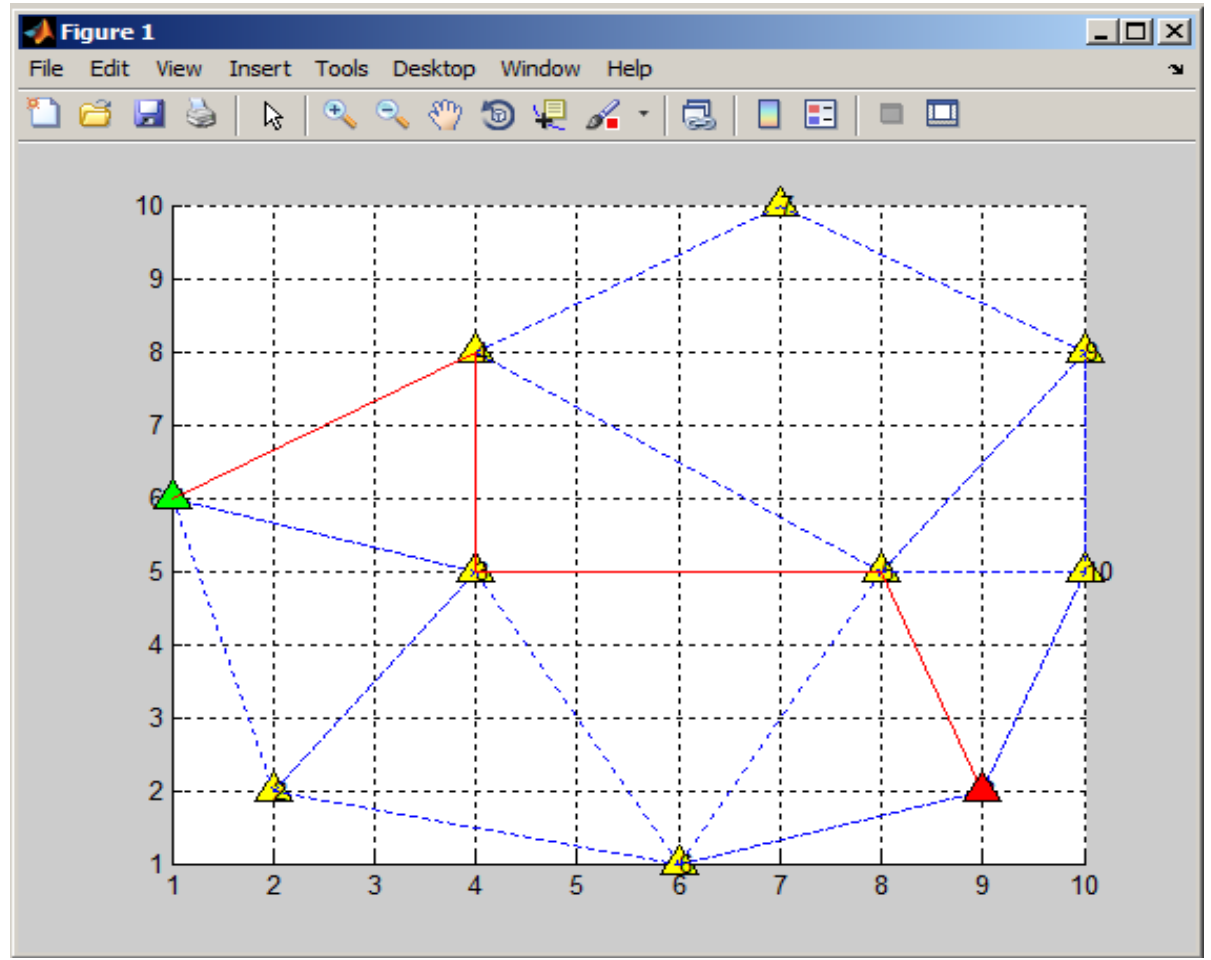

(a) Scenario 1

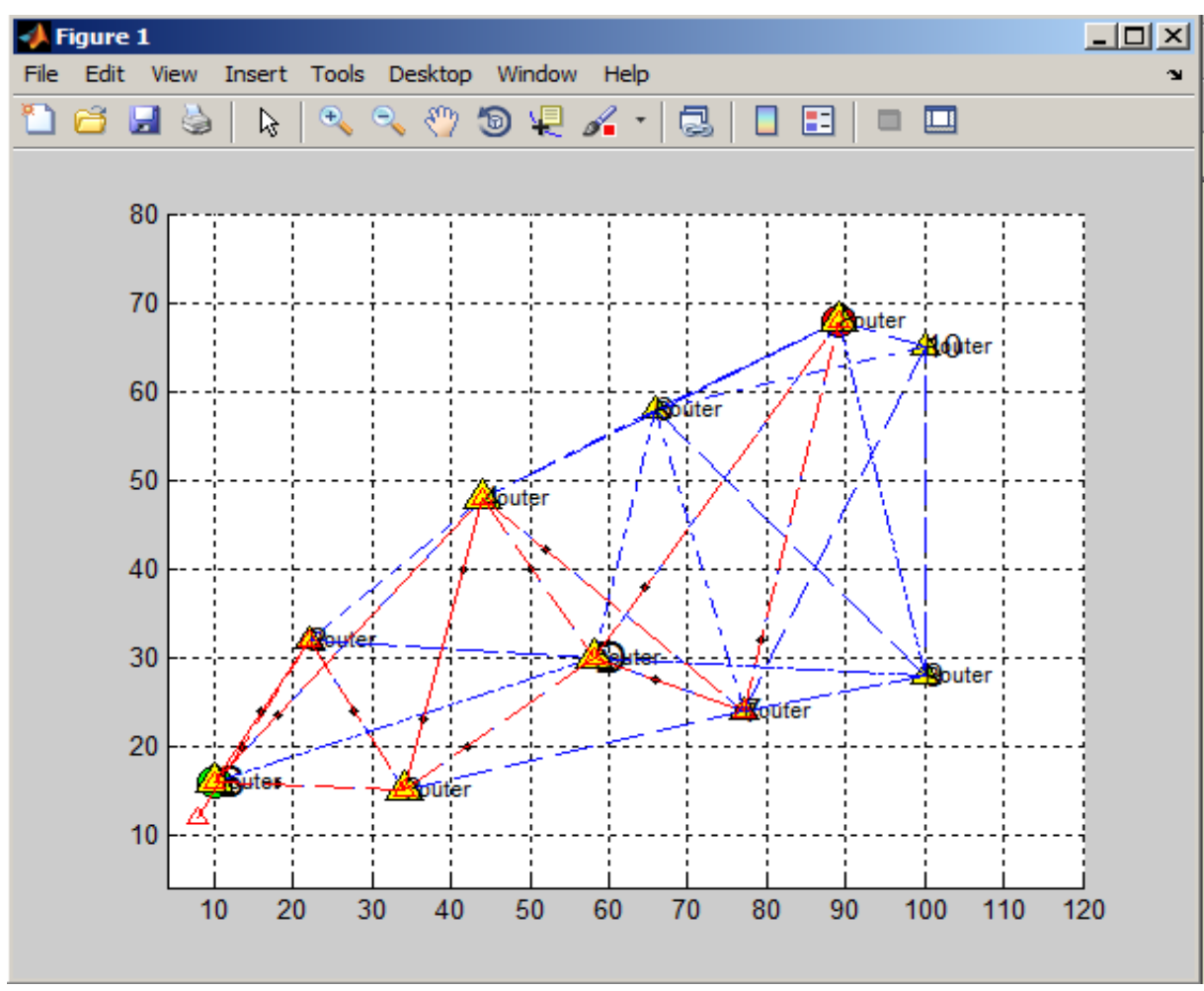

b) Scenario 2 


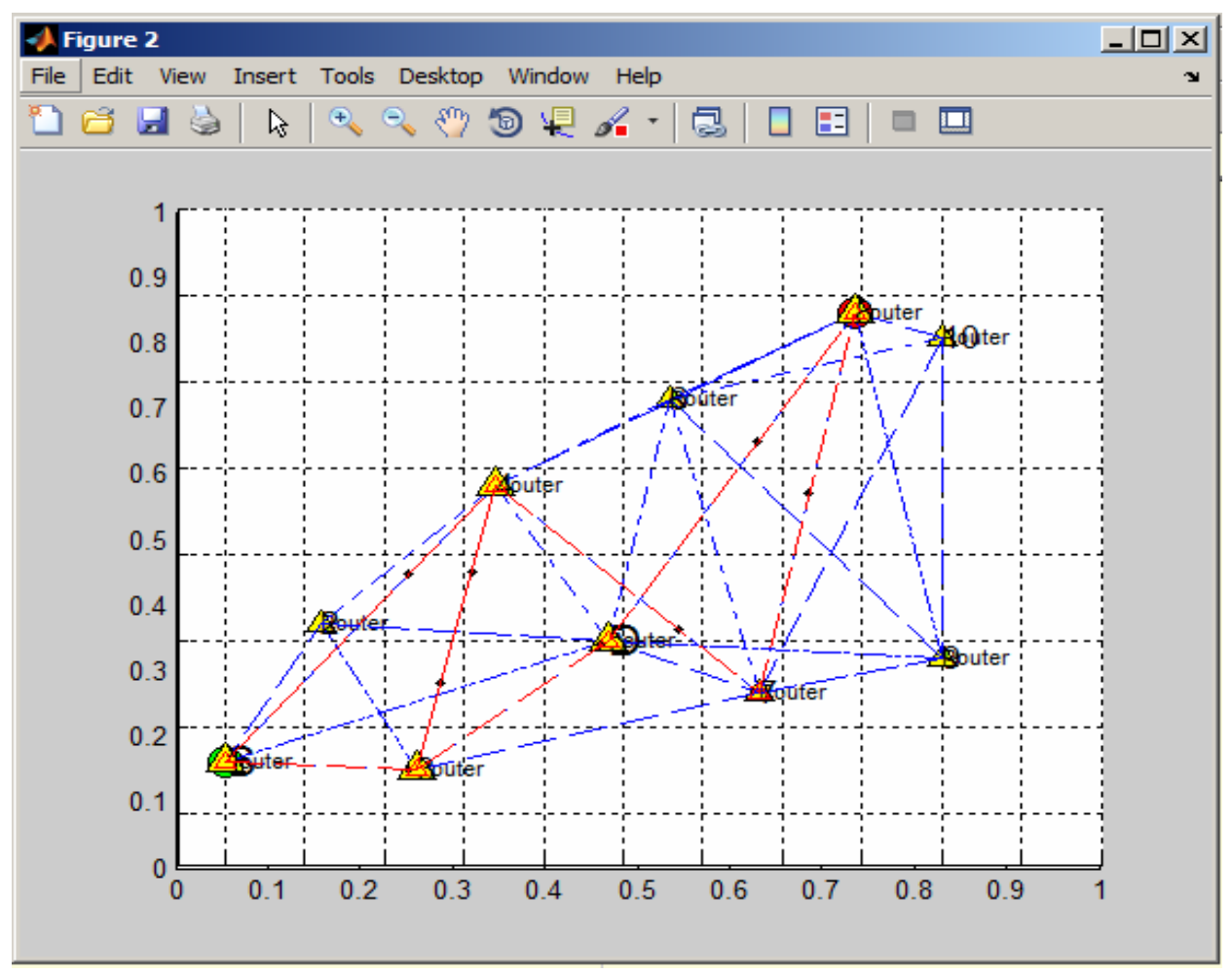

(c) Scenario 3

Figure 5: Simulation scenarios

\section{PERFORMANCE EVALUATION}

To evaluate the performance of our proposed scheme we have performed the simulation using Mat lab. From figure 6 , figure 7, figure 8 and figure 9 we evaluate that with Selective Data Forwarding Scheme (SDFS) the packet drop rate, routing delay and energy consumption are reduced.

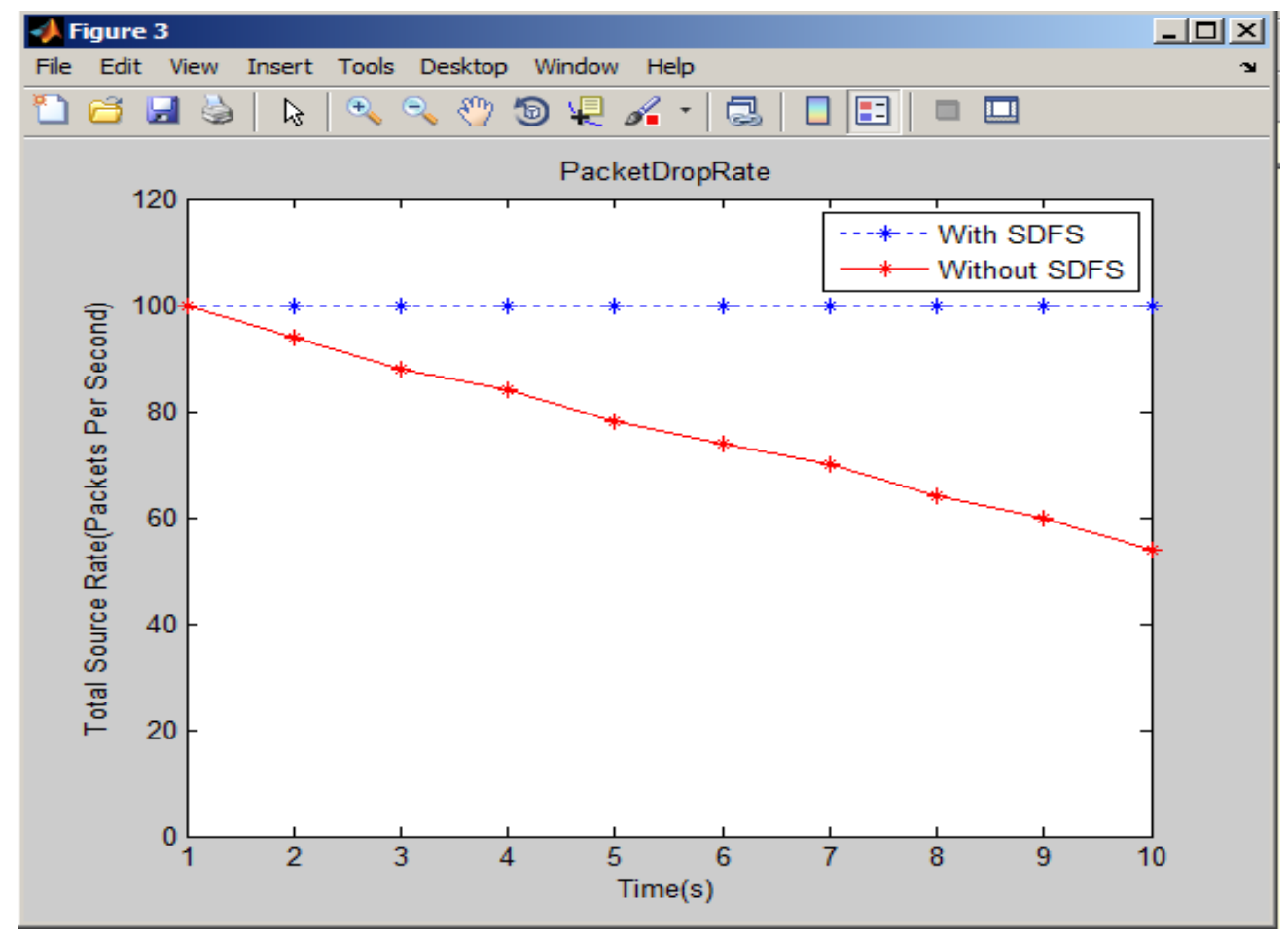

Figure 6: Packet Drop Rate vs Total Source Rate 


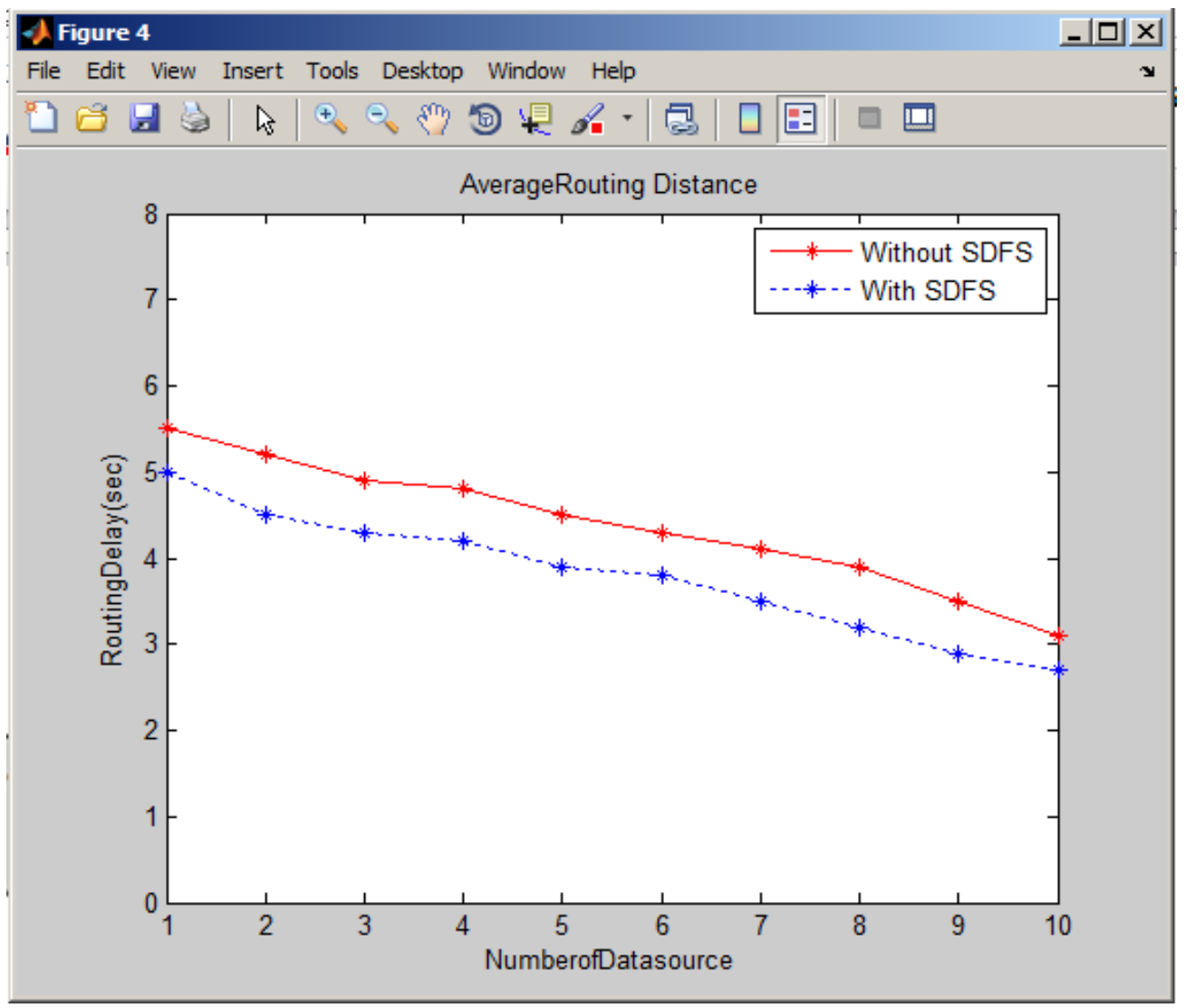

Figure 7: Average Routing Distance vs Routing Delay

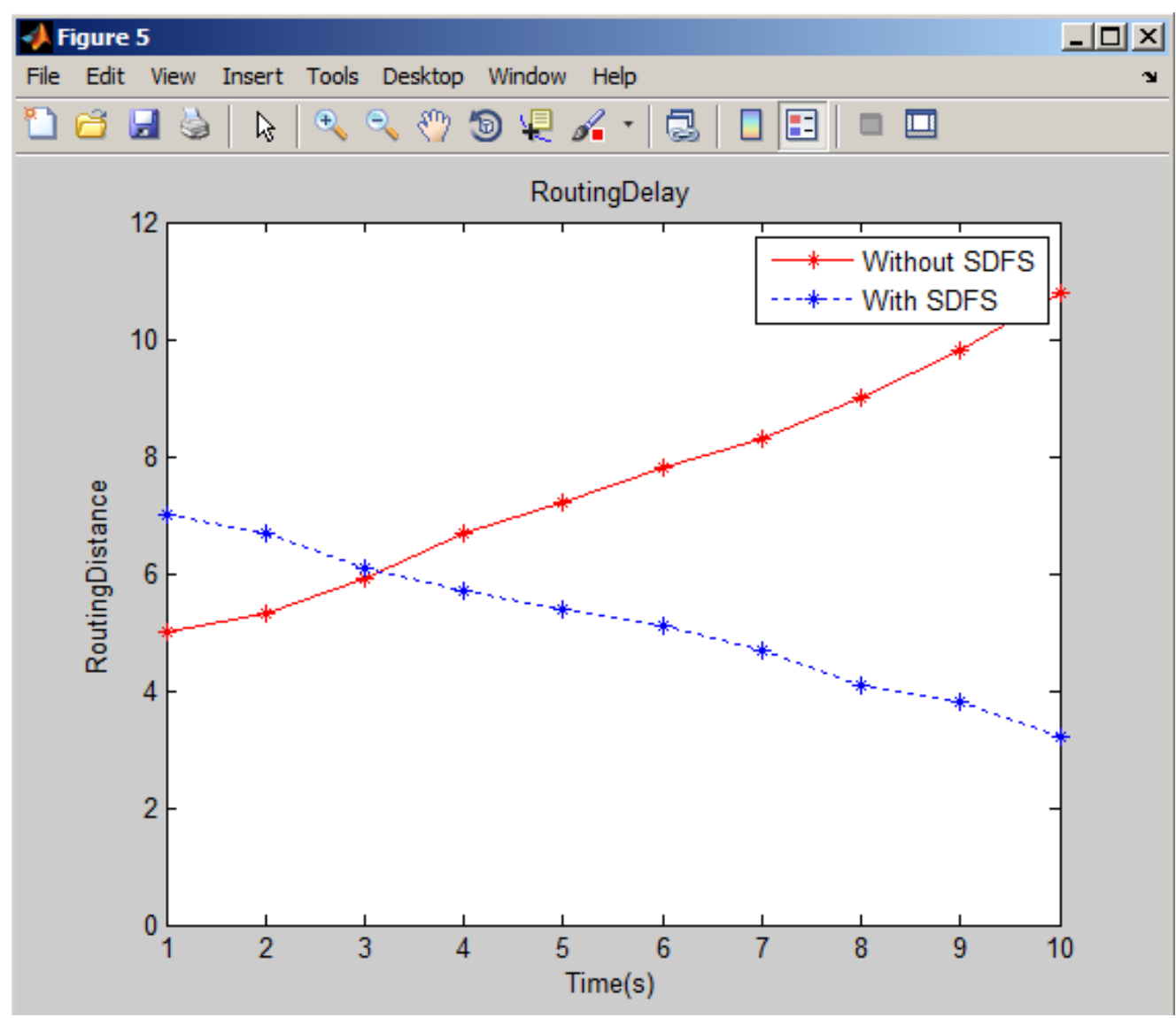

Figure 8: Routing Delay vs Routing Distance 


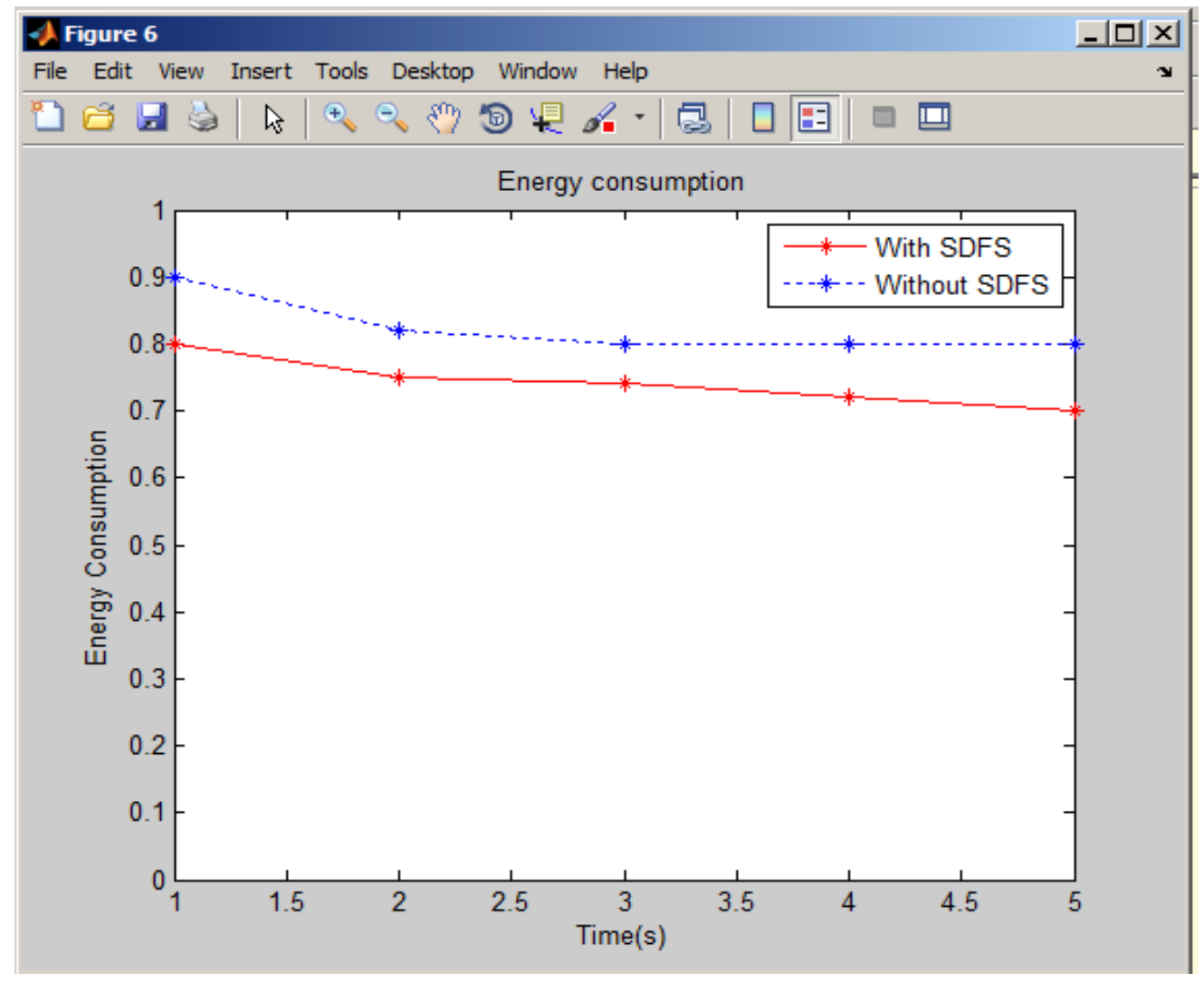

Figure 9: Energy Consumption

\section{COMPARISON OF CONGESTION CONTROL TECHNIQUES}

Table 2 shows the comparison of various congestion control techniques based on, how the congestion is identified, which node is responsible for taking initiative action to control congestion and what is the mechanism used for controlling congestion.

Table 2. Comparison of Congestion Control Techniques

\begin{tabular}{|l|l|l|l|}
\hline \multicolumn{1}{|c|}{ Technique } & \multicolumn{1}{|c|}{$\begin{array}{c}\text { Congestion } \\
\text { Identification }\end{array}$} & \multicolumn{1}{c|}{$\begin{array}{c}\text { Action } \\
\text { Initiator }\end{array}$} & \multicolumn{1}{c|}{$\begin{array}{c}\text { Control } \\
\text { Mechanism }\end{array}$} \\
\hline TADR & Hotspot Node & Source Node & Routing potential fields \\
\hline DPCC & BFS, PCD, DPRA & Source Node & Weighted fair \\
\hline IPD & Buffer Occupancy & Source Node & Assigning priority to the data packets \\
\hline SDFS & Hotspot Node & Source Node & Precedence of data \\
\hline
\end{tabular}

\section{CONCLUSION}

In this paper we proposed the Selective Data Forwarding Scheme (SDFS) to avoid congestion and to optimize the energy drain. This scheme requires information about 1-hop neighbor sensor node to select suitable forward sensor node. For this, each sensor node receives 1-hop CAM periodically from its neighbor sensor node. When sensor node receives 1hop CAM from the neighbor sensor nodes, it should check whether the sensor node is behind, it is in the same path or different path. By using this information each sensor node selects its forward sensor node and maintains the forward table. In the forward table the data is updated using precedence choice rule.

While selecting the forward sensor node, we have to calculate the available energy at the node. If the available energy is superior to the energy required to transmit the data, then the data is transmitted. Otherwise the data is discarded. From simulation results, we can conclude by using the proposed scheme the application's reliability requirements can be satisfied and the congestion in WSN is reduced. 


\section{REFERENCES}

[1] Fengyuan Ren, Member, IEEE, Tao He,Sajal K. Das, Senior Member, IEEE, and Chuang Lin, Senior Member, IEEE'Traffic-Aware Dynamic Routing to Alleviate Congestion in Wireless Sensor Networks", IEEE Transactions on Parallel and Distributed Systems, Vol. 22, No. 9, September 2011

[2] Saeed Rasouli Heikalabad, Ali Ghaffari, Mir Abolgasem Hadian2 and Hossein Rasouli, Technical and Engineering Department, Islamic Azad University Tabriz Branch Tabriz, East Azerbaijan, Iran,"DPCC: Dynamic Predictive Congestion Control in Wireless Sensor Networks", IJCSI International Journal of Computer Science Issues, Vol. 8, Issue 1, January 2011 ISSN (Online): 1694-0814

[3] Rekha Chakravarthi Department of Electronic Sciences Sathyabama University Chennai, India, C.Gomathy Department of Electronics and Control Sathyabama University Chennai,India," IPD: Intelligent Packet Dropping Algorithm for Congestion Control in Wireless Sensor Network", 978-1-4244-9008-0/10/\$26.00 @2010 IEEE

[4] Miguel Sepulcre, Javier Gozalvez, Jerome Harris, and Hanne's Hartenstein," Contextual Communications Congestion Control for Cooperative Vehicular Networks", IEEE transactions on Wireless Communications, vol. 10, no. 2, February 2011.

[5] Do-hyeon Lee, Song-nan Bai, Tae-won Kim; Jae-il Jung," Enhanced Selective Forwarding Scheme for Alert Message Propagation in VANETs", Information Science and Applications (ICISA), 2010 International Conference on April 2010

[6] W.Ye.J. Heinemann and D. Estrin, "An energy-efficient MAC protocol for wireless sensor network", vol.3, June 2002,pp.1567-1576"

[7] Rocio Arroyo-Valles, Antonio G. Marques, and Jesus Cid-Sueiro," Optimal Selective Forwarding for Energy Saving in Wireless Sensor Networks", IEEE Transactions on Wireless Communications, vol. 10, no. 1, January 2011
[8] Rocio Arroyo-Valles, Antonio G. Marques, and Jesus Cid-Sueiro," Energy-efficient Selective Forwarding for Sensor Networks"

[9] Songnan Bai, Zequn Huang, Jaeil Jung," Beacon-based Cooperative Forwarding Scheme for Safety Applications in IVC", INC, IMS and IDC, 2009. NCM '09.Fifth International Joint Conference on Aug. 2009

[10] Zdenek Hanzálek, Member, IEEE, and Petr Jurcík." Energy Efficient Scheduling for ClusterTreeWirelessSensor Networks With Time-Bounded Data Flows: Application to IEEE 802.15.4/ZigBee", IEEE Transactions on Industrial Informatics, vol. 6, no. 3, August 2010

[11] Fengyuan Ren, Tao He, Sajal K. Das, and Chuang Lin," Traffic-Aware Dynamic Routing to Alleviate Congestion in Wireless Sensor Networks", IEEE transactions on Parallel and Distributed Systems, vol. 22, no. 9, September 2011.

[12] Jaewon Kang,, Yanyong Zhang, and Badri Nath, "TARA Topology Aware Resource Adaptation to Alleviate Congestion in Sensor Networks", IEEE Transactions on parallel and distributed systems, vol. 18, no. 7, July 2007

[13] Yung Yi, and Sanjay Shakkottai, "Hop-by-Hop Congestion Control Over a Wireless Multi-Hop Network", IEEE/ACM Transactions on networking, vol. 15, no. 1, February 2007

[14] Md. Mamun-Or-Rashid , Muhammad Mahbub Alam, Md. Abdur Razzaque and Choong Seon Hong "Congestion Avoidance and Fair Event Detection in Wireless Sensor Network",IEICE Transactions on communications, vol, no. , December 2007

[15] Xiaoyan Yin, Xingshe Zhou, Rongsheng Huang,Yuguang Fang, and Shining Li," A FairnessAware Congestion Control Scheme in Wireless Sensor Networks", IEEE Transactions on vehicular Technology, vol. 58, no. 9, November 2009

[16] Fengyuan Ren, Tao He, Sajal K. Das, and Chuang Lin, "Traffic-Aware Dynamic Routing to Alleviate Congestion in Wireless Sensor Networks", IEEE Transactions on parallel and distributed systems, vol. 22, no. 9, September 2011 